TRANSACTIONS OF THE

AMERICAN MATHEMATICAL SOCIETY

Volume 356, Number 7 , Pages 2945-2961

S 0002-9947(03)03393-2

Article electronically published on November 18, 2003

\title{
KOSZUL HOMOLOGY AND EXTREMAL PROPERTIES OF GIN AND LEX
}

\author{
ALDO CONCA
}

\begin{abstract}
For every homogeneous ideal $I$ in a polynomial ring $R$ and for every $p \leq \operatorname{dim} R$ we consider the Koszul homology $H_{i}(p, R / I)$ with respect to a sequence of $p$ of generic linear forms. The Koszul-Betti number $\beta_{i j p}(R / I)$ is, by definition, the dimension of the degree $j$ part of $H_{i}(p, R / I)$. In characteristic 0 , we show that the Koszul-Betti numbers of any ideal $I$ are bounded above by those of the gin-revlex $\operatorname{Gin}(I)$ of $I$ and also by those of the Lex-segment $\operatorname{Lex}(I)$ of $I$. We show that $\beta_{i j p}(R / I)=\beta_{i j p}(R / \operatorname{Gin}(I))$ iff $I$ is componentwise linear and that and $\beta_{i j p}(R / I)=\beta_{i j p}(R / \operatorname{Lex}(I))$ iff $I$ is Gotzmann. We also investigate the set Gins $(I)$ of all the gin of $I$ and show that the Koszul-Betti numbers of any ideal in Gins $(I)$ are bounded below by those of the gin-revlex of $I$. On the other hand, we present examples showing that in general there is no $J$ is $\operatorname{Gins}(I)$ such that the Koszul-Betti numbers of any ideal in Gins $(I)$ are bounded above by those of $J$.
\end{abstract}

\section{INTRODUCTION}

Let $K$ be a field of characteristic 0 and $I$ be a homogeneous ideal of the polynomial ring $R=K\left[x_{1}, \ldots, x_{n}\right]$. We denote by $\beta_{i j}(R / I)$ and by $\beta_{i}(R / I)$ the graded Betti numbers and the total Betti numbers of $R / I$, that is,

$$
\beta_{i j}(R / I)=\operatorname{dim}_{K} \operatorname{Tor}_{i}^{R}(R / I, K)_{j} \quad \text { and } \quad \beta_{i}(R / I)=\operatorname{dim}_{K} \operatorname{Tor}_{i}^{R}(R / I, K),
$$

where the subscript $j$ on the right of a graded module denotes, throughout the paper, the degree $j$ component of that module.

There are two monomial ideals canonically attached to $I$ : the generic initial ideal $\operatorname{Gin}(I)$ with respect to the revlex order and the lex-segment ideal Lex $(I)$. They play a fundamental role in the investigation of many algebraic, homological, combinatorial and geometric properties of the ideal $I$ itself. By definition, the generic initial ideal $\operatorname{Gin}(I)$ is the initial ideal of $I$ with respect to the revlex order after performing a generic change of coordinates. The ideal $\operatorname{Lex}(I)$ is defined as follows. For every vector space $V$ of forms of degree, say, $d$ one defines $\operatorname{Lex}(V)$ to be the vector space generated by the largest, in the lexicographic order, $\operatorname{dim} V$ monomials of degree $d$. For a homogeneous ideal $I$ one sets $\operatorname{Lex}(I)=\bigoplus_{d} \operatorname{Lex}\left(I_{d}\right)$. By the very definition, $\operatorname{Lex}(I)$ is simply a graded vector space, but Macaulay's theorem on Hilbert functions (see for instance [27, Sect.1]) says that $\operatorname{Lex}(I)$ is indeed an ideal. By construction, it is clear that $\operatorname{Lex}(I)$ only depends on the

Received by the editors December 3, 2002 and, in revised form, April 30, 2003.

2000 Mathematics Subject Classification. Primary 13D02; Secondary 13P10, 13Fxx.

Key words and phrases. Koszul homology, Betti numbers, generic initial ideal, Lex-segment. 
Hilbert function of $I$. The graded Betti numbers of $I$, $\operatorname{Gin}(I)$ and $\operatorname{Lex}(I)$ satisfy the following inequalities:

\section{Theorem 1.1.}

(a) $\beta_{i j}(R / I) \leq \beta_{i j}(R / \operatorname{Gin}(I))$ for all $i, j$,

(b) $\beta_{i j}(R / I) \leq \beta_{i j}(R / \operatorname{Lex}(I))$ for all $i, j$.

The first inequality can be proved by a standard deformation argument and holds, more generally, when $\operatorname{Gin}(I)$ is replaced by any initial ideal of $I$, generic or not. The second is an important theorem proved in characteristic 0 by Bigatti 4] and Hulett 21] (independently) and extended later by Pardue [24 to positive characteristic.

Ideals having the same Betti numbers as their generic initial ideal are characterized by the following result of Aramova, Herzog and Hibi [3, Theorem 1.1]:

Theorem 1.2. The following conditions are equivalent:

(a) $\beta_{i j}(R / I)=\beta_{i j}(R / \operatorname{Gin}(I))$ for all $i, j$,

(b) $\beta_{1 j}(R / I)=\beta_{1 j}(R / \operatorname{Gin}(I))$ for all $j$,

(c) $\beta_{1}(R / I)=\beta_{1}(R / \operatorname{Gin}(I))$,

(d) $I$ is componentwise linear.

Conditions (b) and (c) are not explicitly stated in [3] but it follows from the proof that they are indeed equivalent to the others. Similarly, for inequality (b) of Theorem 1.1, Herzog and Hibi [19, Corollary 1.4] proved:

Theorem 1.3. The following conditions are equivalent:

(a) $\beta_{i j}(R / I)=\beta_{i j}(R / \operatorname{Lex}(I))$ for all $i, j$,

(b) $\beta_{1 j}(R / I)=\beta_{1 j}(R / \operatorname{Lex}(I))$ for all $j$,

(c) $\beta_{1}(R / I)=\beta_{1}(R / \operatorname{Lex}(I))$,

(d) $I$ is a Gotzmann ideal.

Recall that a homogeneous ideal $I$ is said to be componentwise linear if for all $k \in \mathbf{N}$ the ideal $I_{\langle k\rangle}$ generated by the elements of degree $k$ of $I$ has a linear resolution. Also, $I$ is said to be a Gotzmann ideal if for every $k \in \mathbf{N}$ the space $I_{k}$ of forms of degree $k$ in $I$ has the smallest possible span in the next degree according to the Macaulay inequality 27, Theorem 3.1], i.e., $\operatorname{dim}_{K} R_{1} I_{k}=\operatorname{dim}_{K} R_{1} \operatorname{Lex}\left(I_{k}\right)$.

Betti numbers can be computed via Koszul homology. Let $K(y, R / I)$ be the graded Koszul complex with values in $R / I$ where $y=y_{1}, \ldots, y_{n}$ is a system of generators of the maximal homogeneous ideal of $R$ and let $H_{i}(y, R / I)$ be its homology. Then

$$
\beta_{i j}(R / I)=\operatorname{dim}_{K} H_{i}(y, R / I)_{j}
$$

More generally, we can consider Koszul homology with respect to a sequence of generic linear forms. We define:

Definition 1.4. For $p=1, \ldots, n$, let $K(p, R / I)$ be the graded Koszul complex with respect to a sequence of $p$ generic linear forms $y_{1}, \ldots, y_{p}$. Denote by $H_{i}(p, R / I)$ the $i$-th homology of $K(p, R / I)$. These are graded modules. We define the Koszul-Betti numbers of $R / I$ by setting

$$
\beta_{i j p}(R / I)=\operatorname{dim}_{K} H_{i}(p, R / I)_{j} .
$$

We extend the definition to the case $p=0$ by setting $H_{0}(0, R / I)=R / I$ and also by setting $H_{i}(p, R / I)=0$ whenever $i>p$. 
We show in this paper that Theorems $1.1,1.2$ and 1.3 hold more generally for Koszul-Betti numbers. This is done in Section 4 while Sections 2 and 3 contain some preliminary results.

In Section 5 we investigate the properties of the set $\operatorname{Gins}(I)$ of all the generic initial ideals of an ideal $I$. We show that the Koszul-Betti numbers of the revlex gin, $\operatorname{Gin}(I)$, are less than or equal to those of any other gin of $I$. On the other hand, we exhibit examples of ideals for which in Gins $(I)$ there is no ideal whose Betti numbers are greater than or equal to those of any other element of Gins $(I)$. To construct these examples we introduce the notion of almost Borel-fixed ideals. The main feature of an almost Borel-fixed ideal $I$ is that the set $\operatorname{Gins}(I)$ can be described in a quite simple way. Then the Eliahou-Kervaire formula for the Betti numbers of Borel-fixed ideals guides us rapidly to the construction of the examples mentioned above. In Section 6 we list some open questions related to the results of Sections 4 ad 5 .

The results, the examples and the open questions presented in this the paper have been inspired and suggested by computations performed by the computer algebra system $\mathrm{CoCoA}[9]$.

\section{Preliminaries}

In this section we introduce some definitions, notation and some preliminary facts. For generalities on term orders, Gröbner bases, initial ideals and lex-segments we refer the reader to [7] 13, 17, 18, 22, 26, 27]. In the following we will consider only term orders $\tau$ such that

$$
x_{1}>_{\tau} x_{2}>_{\tau} \cdots>_{\tau} x_{n} .
$$

By definition, the $\beta_{i j n}(R / I)$ 's are the ordinary graded Betti numbers of $R / I$. For $i=0$ one has $H_{0}(p, R / I)=R / I+\left(y_{1}, \ldots, y_{p}\right)$ and the behavior of its Hilbert function, that is, $\beta_{0 j p}(R / I)$, under Gröbner deformation has been described in [11]. We proved in [11, Theorem 1] that

$$
\beta_{0 j p}(R / I) \leq \beta_{0 j p}\left(R / \operatorname{in}_{\tau}(I)\right)
$$

for all $j$ and $p$ and for all initial ideal $\operatorname{in}_{\tau}(I)$ of $I$. Note also that, since generic linear forms are an almost regular sequence (see [2]), the modules $H_{i}(p, R / I)$ have finite length for all $i>0$ and all $p$. We start with the following:

Lemma 2.1. Let $I, J$ be homogeneous ideals of $R$ and let $\tau$ be a term order. Then

$$
\operatorname{dim}_{K} \operatorname{Tor}_{i}^{R}(R / I, R / J)_{j} \leq \operatorname{dim}_{K} \operatorname{Tor}_{i}^{R}\left(R / \operatorname{in}_{\tau}(I), R / \operatorname{in}_{\tau}(J)\right)_{j}
$$

for all $i$ and $j$.

Proof. The proof follows from a standard deformation argument making use of flat families; see [13, Chap.15] and [25] for details. We will just sketch it. Any vector $\lambda \in \mathbf{R}_{+}^{n}$ induces a graded structure on $R$ and any monomial $m=x_{1}^{\alpha_{1}} \cdots x_{n}^{\alpha_{n}}$ is homogeneous of $\lambda$-degree $\lambda(m)=\sum \alpha_{i} \lambda_{i}$. For every $f \in R$ one defines its $\lambda$-degree to be the the largest degree of a monomial in $f$. If $f \in R$ is a polynomial of $\lambda$-degree $a$, then one defines the initial form $\operatorname{in}_{\lambda}(f)$ of $f$ with respect to $\lambda$ to be the sum of the terms of $f$ of $\lambda$-degree equal to $a$. Let $t$ be a new variable. If $f=\sum \gamma_{i} m_{i} \in R$ with $\gamma_{i} \in K$ and $m_{i}$ monomials and its $\lambda$-degree is $a$, then one defines the $\lambda$-homogenization $\tilde{f}$ of $f$ to be the polynomial $\tilde{f}=\sum \gamma_{i} m_{i} t^{a-\lambda\left(m_{i}\right)}$. Then for every ideal $I$ of $R$ one defines $\operatorname{in}_{\lambda}(I)$ to be the the ideal of $R$ generated 
by $\operatorname{in}_{\lambda}(f)$ with $f \in I$ and $\tilde{I}$ to be the the ideal of $S=R[t]$ generated by $\tilde{f}$ with $f \in I$. By construction one has $S / \tilde{I} \otimes S /(t) \simeq R / \mathrm{in}_{\lambda}(I)$ and one can show that $S / \tilde{I}$ is $K[t]$-free and that $(S / \tilde{I})_{t} \simeq R / I\left[t, t^{-1}\right]$.

Given a term order and a finite set of ideals, $I$ and $J$ in our case, one can represent their initial ideals by means of a weight vector in the sense that there exists $\lambda \in \mathbf{R}_{+}^{n}$ such that $\operatorname{in}_{\lambda}(I)=\operatorname{in}_{\tau}(I)$ an $\operatorname{in}_{\lambda}(J)=\operatorname{in}_{\tau}(J)$. Let $\tilde{I} \subset S=R[t]$ and $\tilde{J} \subset S$ be the $\lambda$-homogenization of $I$ and $J$ with respect to $t$. Consider the bigraded structure on $S$ obtained by giving degree $\left(1, \lambda_{i}\right)$ to $x_{i}$ and degree $(0,1)$ to $t$. By construction $S / \tilde{I}$ and $S / \tilde{J}$ are bigraded $S$-modules and so is $T_{i}=\operatorname{Tor}_{i}^{S}(S / \tilde{I}, S / \tilde{J})$. Let $T_{i j}$ be the direct sum of all the components of $T_{i}$ of bidegree $(j, k)$ as $k$ varies. Since $T_{i j}$ is a finitely generated and graded $K[t]$-module we may decompose it as

$$
T_{i j}=F_{i j} \oplus G_{i j}
$$

where $F_{i j}$ is the free part and $G_{i j}$ is the torsion part which, being $K[t]$-graded, is a direct sum of modules of the form $K[t] /\left(t^{a}\right)$ for various $a>0$. Denote by $t_{i j}$, $f_{i j}$ and $g_{i j}$, respectively, the minimal number of generators of $T_{i j}, F_{i j}$ and $G_{i j}$ as $K[t]$-modules.

Since $t$ is a regular homogeneous element over $S, S / \tilde{I}$ and $S / \tilde{J}$, one has a short exact sequence

$$
0 \rightarrow \operatorname{CoKer} \phi_{i} \rightarrow \operatorname{Tor}_{i}^{R}\left(R / \operatorname{in}_{\tau}(I), R / \operatorname{in}_{\tau}(J)\right) \rightarrow \operatorname{Ker} \phi_{i-1} \rightarrow 0,
$$

where $\phi_{i}$ is the multiplication by $t$ from $T_{i}$ to itself. It follows that the dimension of $\operatorname{Tor}_{i}^{R}\left(R / \operatorname{in}_{\tau}(I), R / \operatorname{in}_{\tau}(J)\right)$ in degree $j$ is given by $t_{i j}+g_{i-1, j}$, while the dimension of $\operatorname{Tor}_{i}^{R}(R / I, R / J)$ in degree $j$ is given by $f_{i j}$. Since $t_{i j}=f_{i j}+g_{i j}$, we are done.

Let $\Delta: \mathbf{R}^{n} \rightarrow \mathbf{R}^{n}$ and $\delta: \mathbf{R}^{n} \rightarrow \mathbf{R}^{n}$ be the linear maps defined by

$$
\begin{aligned}
\Delta(a) & =\left(a_{1}, a_{1}+a_{2}, \ldots, a_{1}+a_{2}+\cdots+a_{n}\right), \\
\delta(a) & =\left(a_{1}-a_{2}, a_{2}-a_{3}, \ldots, a_{n-1}-a_{n}, a_{n}\right) .
\end{aligned}
$$

Fix an integer $d$ and let $M_{d}$ be the set of the monomials of degree $d$ in $R$. The Borel order on $M_{d}$ is defined as follows. Let $x^{a}$ and $x^{b}$ be monomials in $M_{d}$ with exponents $a=\left(a_{1}, \ldots, a_{n}\right)$ and $b=\left(b_{1}, \ldots, b_{n}\right)$. Then

$$
x^{a} \geq_{\text {Borel }} x^{b} \text { iff } \Delta(a) \geq \Delta(b) \text { componentwise. }
$$

The following is a well-known fact:

Lemma 2.2. (1) The Borel order is a partial order on $M_{d}$.

(2) Let $x^{a}$ and $x^{b}$ be monomials of degree $d$. Then $x^{a}>_{\text {Borel }} x^{b}$ iff $x^{a}>_{\tau} x^{b}$ for all term orders $\tau$.

Proof. (1) is easy. To prove (2) one notes that any term order on $M_{d}$ can be represented by a weight function $w=\left(w_{1}, \ldots, w_{n}\right) \in \mathbf{R}^{n}$ with $w_{i}>w_{i+1}$ since we consider only term orders with $x_{i}>x_{i+1}$. Then the desired statement follows immediately from the following identity. For all $a, w \in \mathbf{R}^{n}$ one has

$$
a \cdot w=\Delta(a) \cdot \delta(w)
$$

where $\cdot$ denotes the ordinary scalar product.

A subset $A$ of a poset $P$ is said to be an (upper) poset ideal if for all $a \in A$ and every $b \in P$ with $b>a$ one has $b \in A$. 
Any $g=\left(g_{i j}\right) \in \mathrm{GL}_{n}(K)$ acts on $R$ as a $K$-algebra graded isomorphism by

$$
g\left(x_{i}\right)=\sum_{j=1}^{n} g_{j i} x_{j} .
$$

An ideal $I$ is said to be Borel-fixed if it is invariant under the action of any upper triangular matrix. A monomial ideal $I$ is said to be strongly stable if whenever $x_{i} m$ is in $I$ for some monomial $m$ and some $i$, then $x_{j} m$ is in $I$ for all $j<i$. Equivalently, $I$ is strongly stable iff for every $d$ the set of the monomials of degree $d$ in $I$ form a poset ideal of $M_{d}$ with respect to the Borel order. It turns out that the strongly stable monomial ideals are exactly the Borel-fixed ideals (in positive characteristic this is no longer the case).

For any homogeneous ideal $I$ and any term order $\tau$ one can consider the generic initial ideal $\operatorname{Gin}_{\tau}(I)$ of $I$ with respect to $\tau$, defined as $\operatorname{in}_{\tau}(g(I))$, where $g$ is a generic element in $\mathrm{GL}_{n}(K)$. One has:

Lemma 2.3. Let $I$ be a homogeneous ideal and $\tau$ and $\sigma$ be term orders.

(1) $\operatorname{Gin}_{\tau}(I)$ is a Borel-fixed ideal.

(2) $\operatorname{Gin}_{\tau}(I)=I$ iff $I$ is Borel-fixed.

(3) $\operatorname{Gin}_{\sigma}\left(\operatorname{Gin}_{\tau}(I)\right)=\operatorname{Gin}_{\tau}(I)$.

Proof. (1) is proved, for instance, in [13 Chap.15]. (3) follows from (1) and (2). One direction of implication (2) follows from (1). It remains to prove that $\operatorname{Gin}_{\tau}(I)=I$ for any Borel-fixed ideal $I$. We recall that a generic matrix $g$ has an LU decomposition, that is, it factors as a product $g=a b$, where $b$ is an invertible upper triangular matrix and $a$ is an invertible lower triangular matrix. One can even take one of the two matrices with 1's on the diagonal but we do not mind. This is a well-known theorem in matrix theory and a proof of it with a description of the precise conditions that $g$ must satisfy for having such a decomposition can be found for instance in [16, Theorem 3.2.1]. For every invertible lower triangular matrix $a$ and every monomial $m$ one has

$$
a(m)=\lambda m+\cdots \text { monomials which are }<_{\text {Borel }} m .
$$

It follows that for every polynomial $f$ one has $\operatorname{in}_{\tau}(a(f))=\operatorname{in}_{\tau}(f)$ and hence $\operatorname{in}_{\tau}(a(J))=\operatorname{in}_{\tau}(J)$ for every ideal $J$. Summing up,

$$
\operatorname{Gin}_{\tau}(I)=\operatorname{in}_{\tau}(g(I))=\operatorname{in}_{\tau}(a b(I))=\operatorname{in}_{\tau}(a(I))=\operatorname{in}_{\tau}(I)=I,
$$

where the third equality holds since $b(I)=I$ by assumption and the last holds since $I$ is a monomial ideal.

Lemma 2.4. Let I be a Borel-fixed ideal and let $m_{1}, \ldots, m_{k}$ be its monomial generators. Let $g \in \mathrm{GL}_{n}(K)$ be a generic matrix. Then $g(I)$ is generated by polynomials $f_{1}, \ldots, f_{k}$ of the form $f_{i}=m_{i}+h_{i}$ such that the monomials in $h_{i}$ are smaller than $m_{i}$ in the Borel order. The polynomials $f_{1}, \ldots, f_{k}$ form a Gröbner basis of $g(I)$ with respect to any term order.

Proof. As noted already in the proof of Lemma 2.3 we may write $g=a b$, where $b$ is upper triangular and $a$ is lower triangular. Then $g(I)=a b(I)=a(I)=$ $\left(a\left(m_{1}\right), \ldots, a\left(m_{k}\right)\right)$. Set $f_{i}=\lambda_{i}^{-1} a\left(m_{i}\right)$, where $\lambda_{i}$ is the leading coefficient of $a\left(m_{i}\right)$. By (1) we know that the $f_{i}$ have the desired structure and that $\operatorname{in}_{\tau}(g(I))=$ $\left(\operatorname{in}_{\tau}\left(f_{1}\right), \ldots, \operatorname{in}_{\tau}\left(f_{k}\right)\right)$ follows from Lemma 2.2 
Proper sequences will be of importance in the next sections. Let us recall the definition from [20, Definition 2.1, Remark 2.4]:

Definition 2.5. Let $z_{1}, \ldots, z_{k}$ be a sequence of homogeneous elements in $R$ and let $M$ be a graded $R$-module. Set $I=\left(z_{1}, \ldots, z_{k}\right)$. We say that $z_{1}, \ldots, z_{k}$ is a proper $M$-sequence if one of the following equivalent conditions hold:

1) $z_{j+1} H_{i}\left(z_{1}, \ldots, z_{j}, M\right)=0$ for $j=1, \ldots, k-1$ and $i>0$.

2) $I H_{i}\left(z_{1}, \ldots, z_{j}, M\right)=0$ for $j=1, \ldots, k$ and for $i>0$.

Let $z_{1}, \ldots, z_{k}$ be a proper $M$-sequence, say of linear forms for simplicity. Then the long exact sequence of Koszul homology modules (see [7, Corollary 1.6.13]) splits into shorter ones. Denote by $Z_{j}$ the sequence $z_{1}, \ldots, z_{j}$. For all $i \geq 2$ and all $j \geq 1$ one has

$$
0 \rightarrow H_{i}\left(Z_{j}, M\right) \rightarrow H_{i}\left(Z_{j+1}, M\right) \rightarrow H_{i-1}\left(Z_{j}, M\right)(-1) \rightarrow 0
$$

and

$$
\begin{aligned}
0 \rightarrow H_{1}\left(Z_{j}, M\right) & \rightarrow H_{1}\left(Z_{j+1}, M\right) \\
& \rightarrow H_{0}\left(Z_{j}, M\right)(-1) \stackrel{\bullet}{\longrightarrow} H_{0}\left(Z_{j}, M\right) \rightarrow H_{0}\left(Z_{j+1}, M\right) \rightarrow 0,
\end{aligned}
$$

where the map $\bullet$ is the multiplication by $z_{j+1}$ (up to sign).

\section{Koszul-Betti numbers And Borel-FiXed ideAls}

First of all, we explain why the last $p$ variables are "generic" for a Borel-fixed ideal.

Lemma 3.1. Let $I$ be a Borel-fixed ideal. For a given $p, 1 \leq p \leq n$, let $X_{p}$ be the sequence $x_{n-p+1}, x_{n-p+2}, \ldots, x_{n}$. Then $\beta_{i j p}(R / I)=\operatorname{dim}_{K} H_{i}\left(X_{p}, R / I\right)_{j}$.

Proof. Let $y=y_{1}, \ldots, y_{p}$ be a sequence of $p$ generic linear forms. We can choose an upper-triangular matrix $g$ such that the induced $K$-algebra graded isomorphism $g: R \rightarrow R$ maps the linear space generated by $y$ to the linear space generated by $X_{p}$. Then $H_{i}(y, R / I)=H_{i}\left(X_{p}, R / g(I)\right)$ and $g(I)=I$ by assumption.

For a Borel-fixed ideal $I$ a formula for the Koszul-Betti numbers of $R / I$ has been described by Aramova and Herzog [1 Proposition 2.1]. Their result holds more generally for a stable ideal and can be seen as an extension of the EliahouKervaire [14] formula for the Betti numbers. To state their formula in the form which best suits our needs we introduce a useful piece of notation. For a monomial $u$ with exponent $a=\left(a_{1}, \ldots, a_{n}\right)$ one defines

$$
\max (u)=\max \left\{i: a_{i}>0\right\} .
$$

For a set of monomials $A$ and for $i=1, \ldots, n$ we put

$$
\begin{aligned}
m_{i}(A) & =|\{u \in A: \max (u)=i\}|, \\
m_{\leq i}(A) & =|\{u \in A: \max (u) \leq i\}|, \\
m_{i j}(A) & =\mid\{u \in A: \max (u)=i \text { and } \operatorname{deg}(u)=j\} \mid .
\end{aligned}
$$

When $I$ is either a vector space generated by monomials of the same degree or a monomial ideal, we set

$$
m_{i}(I)=m_{i}(G), \quad m_{\leq i}(I)=m_{\leq i}(G), \quad m_{i j}(I)=m_{i j}(G),
$$

where $G$ is the set of the minimal monomial (vector space or ideal) generators of $I$. 
Taking into consideration Lemma 3.1, the result of Aramova and Herzog [1] can be stated as follows:

Theorem 3.2 (Aramova-Herzog). Let $I$ be a Borel-fixed ideal. Then for all $i>0$ and for all $j$ and $p$ the Koszul-Betti numbers of $R / I$ are given by the formula

$$
\beta_{i j p}(R / I)=\sum_{s=i+n-p}^{n} m_{s, j-i+1}(I)\left(\begin{array}{c}
s+p-n-1 \\
i-1
\end{array}\right) .
$$

With $p=n$ one gets the Eliahou-Kervaire formula for Betti numbers:

Theorem 3.3 (Eliahou-Kervaire). Let I be a Borel-fixed ideal. Then for all $i>0$ and for all $j$ the Betti numbers of $R / I$ are given by the formula

$$
\beta_{i j}(R / I)=\sum_{s=i}^{n} m_{s, j-i+1}(I)\left(\begin{array}{c}
s-1 \\
i-1
\end{array}\right) .
$$

We also have:

Lemma 3.4. Let I be a Borel-fixed ideal. Then for all $j$ and $p$ one has

$$
\beta_{0 j p}(R / I)=\left(\begin{array}{c}
n-p-1+j \\
n-p-1
\end{array}\right)-m_{\leq n-p}\left(I_{j}\right) .
$$

Proof. This follows immediately from the definition of $m_{\leq i}$ since, by Lemma 3.1 the sequence $x_{n-p+1}, x_{n-p+2}, \ldots, x_{n}$ is generic for $I$.

The following is a useful property of Borel-fixed spaces; see [4, Proposition 1.3].

Proposition 3.5. Let $B$ be a Borel-fixed vector space of monomials of degree $d$. Then one has $m_{i}\left(R_{1} B\right)=m_{\leq i}(B)$.

The functions $m_{i}$ and $m_{\leq i}$ are important for comparing Borel-fixed ideals. Here is why:

Proposition 3.6. Let $I, J$ be Borel-fixed ideals with the same Hilbert function. Assume that $m_{\leq i}\left(J_{j}\right) \leq m_{\leq i}\left(I_{j}\right)$ for all $i$ and $j$. Then one has:

i) $m_{i}(I) \leq m_{i}(J)$ for all $i$.

ii) $\beta_{i j p}(R / I) \leq \beta_{i j p}(R / J)$ for all $i, j, p$.

Proof. i) Let $k$ be an integer such that both $I$ and $J$ are generated in degree $\leq k$. The ideals $I_{\langle k\rangle}$ and $J_{\langle k\rangle}$ are Borel-fixed generated in degree $k$ and have the same Hilbert function. By the Eliahou-Kervaire formula, they both have a linear resolution. It follows that the Betti numbers of $I_{\langle k\rangle}$ and $J_{\langle k\rangle}$ are the same. Again by the Eliahou-Kervaire formula it follows that $m_{i}\left(I_{k}\right)=m_{i}\left(J_{k}\right)$ for all $i$ and hence $m_{\leq i}\left(I_{k}\right)=m_{\leq i}\left(J_{k}\right)$ for all $i$. Now note that we have $m_{i}(I)=\sum_{j=1}^{k} m_{i j}(I)$ and $m_{i j}(I)=m_{i}\left(I_{j}\right)-m_{i}\left(R_{1} I_{j-1}\right)$. We may write $m_{i}\left(I_{j}\right)=m_{\leq i}\left(I_{j}\right)-m_{\leq i-1}\left(I_{j}\right)$ and by Proposition 3.5 we have $m_{i}\left(R_{1} I_{j-1}\right)=m_{\leq i}\left(I_{j-1}\right)$. Summing up, we have

$$
m_{i}(I)=\sum_{j=1}^{k}\left[m_{\leq i}\left(I_{j}\right)-m_{\leq i-1}\left(I_{j}\right)-m_{\leq i}\left(I_{j-1}\right)\right]=m_{\leq i}\left(I_{k}\right)-\sum_{j=1}^{k} m_{\leq i-1}\left(I_{j}\right) .
$$

The same formula also holds when we replace $I$ with $J$. Since we know that $m_{\leq i}\left(I_{k}\right)=m_{\leq i}\left(J_{k}\right)$, it follows that

$$
m_{i}(J)-m_{i}(I)=\sum_{j \geq 1}\left[m_{\leq i-1}\left(I_{j}\right)-m_{\leq i-1}\left(J_{j}\right)\right] .
$$


By assumption $m_{\leq i-1}\left(I_{j}\right)-m_{\leq i-1}\left(J_{j}\right)$ is non-negative and hence we may conclude that $m_{i}(J) \geq m_{i}(I)$.

ii) By virtue of Lemma 3.4 for $i=0$ one has

$$
\beta_{0 j p}(R / J)-\beta_{0 j p}(R / I)=m_{\leq n-p}\left(I_{j}\right)-m_{\leq n-p}\left(J_{j}\right) .
$$

Then $\beta_{0 j p}(R / I) \leq \beta_{0 j p}(R / J)$ follows from the assumption. To prove the inequality for $i>0$ we just follows Bigatti's proof of Theorem 1.1(b) by replacing the EliahouKervaire formula with the Aramova-Herzog formula. For the reader's convenience we reproduce it here. First, by Proposition 3.5, in the Aramova-Herzog formula we may replace $m_{s, j-i+1}(I)$ with $m_{s}\left(I_{j-i+1}\right)-m_{\leq s}\left(I_{j-i}\right)$. Then we may replace $m_{s}\left(I_{j-i+1}\right)$ with $m_{\leq s}\left(I_{j-i+1}\right)-m_{\leq s-1}\left(I_{j-i+1}\right)$. We get

$\beta_{i j p}(R / I)=\sum_{s=i+n-p}^{n}\left[m_{\leq s}\left(I_{j-i+1}\right)-m_{\leq s-1}\left(I_{j-i+1}\right)-m_{\leq s}\left(I_{j-i}\right)\right]\left(\begin{array}{c}s+p-n-1 \\ i-1\end{array}\right)$

which we can rewrite as a sum of three terms:

$$
\begin{gathered}
m_{\leq n}\left(I_{j-i+1}\right)\left(\begin{array}{c}
p-1 \\
i-1
\end{array}\right)-m_{\leq n-p+i-1}\left(I_{j-i+1}\right), \\
\sum_{s=i+n-p}^{n-1} m_{\leq s}\left(I_{j-i+1}\right)\left[\left(\begin{array}{c}
s+p-n-1 \\
i-1
\end{array}\right)-\left(\begin{array}{c}
s+p-n \\
i-1
\end{array}\right)\right], \\
-\sum_{s=i+n-p}^{n} m_{\leq s}\left(I_{j-i}\right)\left(\begin{array}{c}
s+p-n-1 \\
i-1
\end{array}\right) .
\end{gathered}
$$

Note that $m_{\leq n}\left(I_{j-i+1}\right)=\operatorname{dim}_{K}\left(I_{j-i+1}\right)$ and hence (i) can be rewritten as

$$
\operatorname{dim}_{K}\left(I_{j-i+1}\right)\left(\begin{array}{c}
p-1 \\
i-1
\end{array}\right)-m_{\leq n-p+i-1}\left(I_{j-i+1}\right) .
$$

Also, applying Pascal's triangle formula, part (ii) is equal to

$$
-\sum_{s=i+n-p}^{n-1} m_{\leq s}\left(I_{j-i+1}\right)\left(\begin{array}{c}
s+p-n-1 \\
i-2
\end{array}\right) .
$$

Summing up, $\beta_{i j p}(R / I)$ is the sum of (iii), (iv) and (v). The crucial consequence is that $\beta_{i j p}(R / I)$ can be written as $\operatorname{dim}_{K}\left(I_{j-i+1}\right)\left(\begin{array}{c}p-1 \\ i-1\end{array}\right)$ minus a linear combination with non-negative coefficients of $m_{\leq a}\left(I_{b}\right)$ for various $a$ and $b$. $\operatorname{Since} \operatorname{dim}_{K}\left(I_{j-i+1}\right)=$ $\operatorname{dim}_{K}\left(J_{j-i+1}\right)$, it follows that $\beta_{i j p}(R / J)-\beta_{i j p}(R / I)$ can be written as a linear combination with non-negative coefficients of $m_{\leq a}\left(I_{b}\right)-m_{\leq a}\left(J_{b}\right)$. By assumption the latter is non-negative and we conclude that $\beta_{i j p}(R / J)-\beta_{i j p}(R / I) \geq 0$.

Proposition 3.7. Let $I, J$ be Borel-fixed ideals with the same Hilbert function. Assume that $m_{\leq i}\left(J_{j}\right) \leq m_{\leq i}\left(I_{j}\right)$ for all $i$ and $j$. Then the following conditions are equivalent:

i) $\beta_{i j p}(R / I)=\beta_{i j p}(R / J)$ for all $i, j$ and $p$,

ii) $\beta_{i j}(R / I)=\beta_{i j}(R / J)$ for all $i$ and $j$,

iii) $\beta_{1 j}(R / I)=\beta_{1 j}(R / J)$ for all $j$,

iv) $\beta_{1}(R / I)=\beta_{1}(R / J)$,

v) $m_{i j}(I)=m_{i j}(J)$ for all $i, j$. 
vi) $m_{i}(I)=m_{i}(J)$ for all $i$.

vii) $m_{i}\left(I_{j}\right)=m_{i}\left(J_{j}\right)$ for all $i$ and $j$.

viii) $m_{\leq i}\left(I_{j}\right)=m_{\leq i}\left(J_{j}\right)$ for all $i$ and $j$.

Proof. The implications i) $\Rightarrow$ ii) $\Rightarrow$ iii) $\Rightarrow$ iv) and v) $\Rightarrow$ vi) are obvious. That i) $\Leftrightarrow$ viii) and vi) $\Leftrightarrow$ viii) follow from the proof of Proposition 3.6 . Also iv) $\Rightarrow$ vi) follows from Proposition 3.6 since $\beta_{1}(R / I)=\sum m_{i}(I)$. Furthermore vii) $\Leftrightarrow$ viii) is easy and vii), viii) $\Rightarrow \mathrm{v}$ ) since, by Proposition 3.5 we have $m_{i j}(I)=m_{i}\left(I_{j}\right)-$ $m_{i}\left(R_{1} I_{j-1}\right)=m_{i}\left(I_{j}\right)-m_{\leq i}\left(I_{j-1}\right)$.

\section{Koszul-Betti numbers, Gin And Lex}

In this section we prove the main results of this note. To compare Betti numbers of Borel-fixed and lex-segment ideals Bigatti made a study of the behavior of the functions $m_{i}(\ldots)$ as one compares a Borel-fixed space with its lex-segment. She proved in [4, Theorem 2.1] the following crucial inequality. An essentially equivalent form of the same result appears also in D. Bayer's Ph.D. thesis [5, Lemma 8.1].

Proposition 4.1. Let $I$ be a Borel-fixed ideal and let $L=\operatorname{Lex}(I)$ be the corresponding lex-segment. Then one has $m_{\leq i}\left(L_{j}\right) \leq m_{\leq i}\left(I_{j}\right)$ for all $i$ and $j$.

Remark/Example 4.2. a) With the notation and the assumption of Proposition 3.6, it is not true in general that $m_{i j}(I) \leq m_{i j}(J)$ for all $i, j$. For instance take $I=\left(x_{1}, x_{2}\right)^{2}+\left(x_{1}, x_{2}, x_{3}\right)^{3}$. The ideal $I$ is Borel-fixed, and its lex-segment ideal is $L=x_{1}\left(x_{1}, x_{2}, x_{3}\right)+\left(x_{1}, x_{2}, x_{3}\right)^{3}$. By Proposition 4.1 we know that $m_{\leq i}\left(L_{j}\right) \leq$ $m_{\leq i}\left(I_{j}\right)$ for all $i, j$ but $m_{2,2}(I)=2$ and $m_{2,2}(L)=1$.

b) By Propositions [3.7 and 4.1 we know that a Borel-fixed ideal $I$ with lexsegment ideal $L$ is Gotzmann iff $m_{i}(I)=m_{i}(L)$ for all $i$. This is an example, perhaps the smallest one, of a Borel-fixed Gotzmann ideal $I$ which is not a lexsegment ideal. In $K\left[x_{1}, x_{2}, x_{3}\right]$ consider

$$
I=\left(x_{1}^{3}, x_{1}^{2} x_{2}, x_{1}^{2} x_{3}, x_{1} x_{2}^{2}, x_{1} x_{2} x_{3}, x_{2}^{3}, x_{2}^{2} x_{3}\right) .
$$

Its lex-segment ideal is

$$
L=\left(x_{1}^{3}, x_{1}^{2} x_{2}, x_{1}^{2} x_{3}, x_{1} x_{2}^{2}, x_{1} x_{2} x_{3}, x_{1} x_{3}^{2}, x_{2}^{3}\right) .
$$

To verify that $I$ is Gotzmann it suffices to note that $m_{1}(I)=m_{1}(L)=1, m_{2}(I)=$ $m_{2}(L)=2$ and $m_{3}(I)=m_{3}(L)=4$.

This is the generalization of Theorem [1.1:

Theorem 4.3. Let $\tau$ be any term order and $I$ a homogeneous ideal. Then

a) $\beta_{i j p}(R / I) \leq \beta_{i j p}\left(R / \operatorname{Gin}_{\tau}(I)\right)$ for all $i, j, p$.

b) $\beta_{i j p}(R / I) \leq \beta_{i j p}(R / \operatorname{Lex}(I))$ for all $i, j, p$.

Proof. Let $y=y_{1}, \ldots, y_{p}$ be a sequence of generic linear forms. Let $g \in \mathrm{GL}_{n}(K)$ such that the induced $K$-algebra graded isomorphism $g: R \rightarrow R$ maps $y_{i}$ to $x_{n-p+i}$ for $i=1, \ldots, p$. Denote by $X_{p}$ the sequence $x_{n-p+1}, x_{n-p+2}, \ldots, x_{n}$. Then

$$
H_{i}(y, R / I)=H_{i}\left(X_{p}, R / g(I)\right)=\operatorname{Tor}_{i}^{R}\left(R / g(I), R /\left(X_{p}\right)\right) .
$$

It follows by Lemma 2.1 that

$$
\beta_{i j p}(R / I) \leq \operatorname{dim}_{K} \operatorname{Tor}_{i}^{R}\left(R / \operatorname{in}_{\tau}(g(I)), R /\left(X_{p}\right)\right)_{j}=\operatorname{dim}_{K} H_{i}\left(X_{p}, R / \operatorname{in}_{\tau}(g(I))_{j} .\right.
$$


Since the $y_{i}$ are generic, $g$ can be chosen generic as well. So $\operatorname{in}_{\tau}(g(I))=\operatorname{Gin}_{\tau}(I)$ and it is Borel-fixed. But then by Lemma 3.1] we know that

$$
\beta_{i j p}\left(R / \operatorname{Gin}_{\tau}(I)\right)=H_{i}\left(X_{p}, R / \operatorname{Gin}_{\tau}(I)\right)_{j}
$$

and this proves a).

To prove b), by virtue of a), we may replace $I$ with its gin, that is, we may assume that $I$ is Borel-fixed. Then the result follows from Propositions 3.6 and 4.1 .

Remark 4.4. a) Theorem 4.3 a) also holds in positive characteristic.

b) It is easy to see that for any ideal $I$ one has $\beta_{0 j p}(R / I)=\beta_{0 j p}(R / \operatorname{Gin}(I))$ for all $j$ and $p$; see [11, Lemma 2] for details.

Now we generalize Theorem 1.2

Theorem 4.5. The following conditions are equivalent:

i) $\beta_{i j p}(R / I)=\beta_{i j p}(R / \operatorname{Gin}(I))$ for all $i, j, p$.

ii) $\beta_{1 j n}(R / I)=\beta_{1 j n}(R / \operatorname{Gin}(I))$ for all $j$.

iii) $I$ is a componentwise linear ideal.

iv) A generic sequence of linear forms $y_{1}, \ldots, y_{n}$ is a proper sequence over $R / I$.

Proof. i) $\Rightarrow$ ii) is obvious and ii) $\Rightarrow$ iii) holds by Theorem 1.2

We now prove that iii) $\Rightarrow$ iv). Assuming that $I$ is componentwise linear we have to show that $\mathbf{m} H_{i}(p, R / I)=0$ for all $i>0$ and $p$, where $\mathbf{m}$ denotes the homogeneous maximal ideal of $R$. First note that if $I$ is generated in a single degree, say $d$, then so does $\operatorname{Gin}(I)$. Then by Theorem 3.2 we have that for $i>0$ the homology module $H_{i}(p, R / \operatorname{Gin}(I))$ is concentrated in a single degree, namely $d+i-$ 1. By Theorem4.3 the same is true also for $H_{i}(p, R / I)$ and hence $\mathbf{m} H_{i}(p, R / I)=0$. Now assume that $I$ is possibly generated in distinct degrees. For a fixed $p$, let $K_{\bullet}=K(p, R)$ be the Koszul complex of $p$ generic linear forms and let $\phi_{i}$ be the map from $K_{i}$ to $K_{i-1}$. Let $a \in H_{i}(p, R / I)$ be a homogeneous element, say of degree $s$. We may represent $a$ as a residue class, say $a=\bar{f}$, of an element $f \in K_{i}$. Then $\phi_{i}(f)$ is in $I K_{i-1}$. Since $\phi_{i}(f)$ is homogeneous of degree $s$, we deduce that $\phi_{i}(f)$ is in $I_{k} K_{i-1}$ where $k=s-i+1$. Set $J=I_{\langle k\rangle}$, that is, $J$ is the ideal generated by $I_{k}$. We have that the class of $f$ is in $H_{i}(p, R / J)$. By construction $J$ is generated in a single degree and by assumption it has a linear resolution. From what we have seen above we may conclude that $\mathbf{m} f$ is contained in Image $\phi_{i+1}+J K_{i}$. Since $J \subset I$ we have that $\mathbf{m} f$ is contained in Image $\phi_{i+1}+I K_{i}$ which in turn implies that $\mathbf{m} a=0$ in $H_{i}(p, R / I)$.

It remains to prove that iv) implies i). We have already mentioned in Remark 4.4 that $\beta_{0 j p}(R / I)=\beta_{0 j p}(R / \operatorname{Gin}(I))$ for all $j$ and $p$ holds for any ideal $I$. Assuming iv), it is then enough to show that the numbers $\beta_{i j p}(R / I)$ only depend on the numbers $\beta_{0 j p}(R / I)$. For $i=1$ the exact sequence (2) implies that

$$
\beta_{1 j p}(R / I)=\beta_{1 j p-1}(R / I)+\beta_{0 j-1 p-1}(R / I)-\beta_{0 j p-1}(R / I)+\beta_{0 j p}(R / I) .
$$

For $i>1$ the exact sequence (3) implies that

$$
\beta_{i j p}(R / I)=\beta_{i j p-1}(R / I)+\beta_{i-1 j-1 p-1}(R / I) .
$$

Since $\beta_{i j p}(R / I)=0$ for all $i>p$ these recursive relations imply what we want and conclude the proof. 
Now we generalize Theorem 1.3

Theorem 4.6. The following conditions are equivalent:

i) $\beta_{i j p}(R / I)=\beta_{i j p}(R / \operatorname{Lex}(I))$ for all $i, j, p$.

ii) $\beta_{1 j n}(R / I)=\beta_{1 j n}(R / \operatorname{Lex}(I))$ for all $j$.

iii) $I$ is a Gotzmann ideal.

iv) $\beta_{0 j p}(R / I)=\beta_{0 j p}(R / \operatorname{Lex}(I))$ for all $j, p$ and $I$ is componentwise linear.

Proof. Set $L=\operatorname{Lex}(I)$. i) $\Rightarrow$ ii) is obvious and ii) $\Rightarrow$ iii) holds by Theorem 1.3. A Gotzmann ideal is clearly componentwise linear. So to prove that iii) implies iv), it suffices to prove that $\beta_{0 j p}(R / I)=\beta_{0 j p}(R / L)$ for all $j, p$. By Remark 4.4 passing to the gin does not change $\beta_{0 j p}$. So we may replace $I$ with $\operatorname{Gin}(I)$ which is still Gotzmann. In other words we may assume that $I$ is Borel-fixed and $\beta_{1}(R / I)=$ $\beta_{1}(R / L)$. Then by Propositions 4.1 and 3.7 we know that $\beta_{0 j p}(R / I)=\beta_{0 j p}(R / L)$ for all $j, p$. Finally, we prove that iv) implies i). Since $I$ is componentwise linear, by virtue of Theorem 4.5, we may replace $I$ with its gin, that is, we may assume that $I$ is Borel-fixed. The assumption $\beta_{0 j p}(R / I)=\beta_{0 j p}(R / L)$, by virtue of Lemma 3.4. translates into $m_{\leq i}\left(I_{j}\right)=m_{\leq i}\left(L_{j}\right)$ for all $i$ and $j$. By Propositions 3.7 and 4.1 this implies that $\beta_{i j p}(R / I)=\beta_{i j p}(R / L)$ for all $i, j, p$.

\section{COMPARING THE Gins}

Given a homogeneous ideal $I$ we may consider the set

$$
\operatorname{Gins}(I)=\left\{\operatorname{Gin}_{\tau}(I): \tau \text { is a term order }\right\}
$$

of all the generic initial ideals of $I$. Among them, the gin-revlex (which we simply denote by $\operatorname{Gin}(I))$ plays a special role. For instance, it is known that $\operatorname{projdim}(I)=$ $\operatorname{projdim}(\operatorname{Gin}(I))$ and $\operatorname{reg}(I)=\operatorname{reg}(\operatorname{Gin}(I))$, where projdim and reg denote the projective dimension and the Castelnuovo-Mumford regularity; see [6]. It follows that

$$
\operatorname{projdim}(\operatorname{Gin}(I)) \leq \operatorname{projdim}\left(\operatorname{Gin}_{\tau}(I)\right) \quad \text { and } \quad \operatorname{reg}(\operatorname{Gin}(I)) \leq \operatorname{reg}\left(\operatorname{Gin}_{\tau}(I)\right)
$$

for every term order $\tau$. We generalize this and show that:

Theorem 5.1. Let I be a homogeneous ideal. Let $\tau$ be a term order. Then

$$
\beta_{i j p}(\operatorname{Gin}(I)) \leq \beta_{i j p}\left(\operatorname{Gin}_{\tau}(I)\right)
$$

for every $i, j, p$.

Proof. Set $J=\operatorname{Gin}(I)$ and $H=\operatorname{Gin}_{\tau}(I)$. Note that $J$ and $H$ are Borel-fixed ideals with the same Hilbert function. By virtue of Proposition 3.6] it is therefore enough to show that $m_{\leq i}\left(H_{j}\right) \leq m_{\leq i}\left(J_{j}\right)$ for all $i$ and $j$. Let $a_{1}, \ldots, a_{k}$ be the generators of $J_{j}$ and $b_{1}, \ldots, b_{k}$ that of $H_{j}$. We may order the $a_{r}$ 's and the $b_{r}$ 's according to the revlex order. We claim the following:

Claim. $a_{r} \geq b_{r}$ in the revlex order for all $r$.

This implies that $\max \left(a_{r}\right) \leq \max \left(b_{r}\right)$ for all $r$ and hence $m_{\leq i}\left(H_{j}\right) \leq m_{\leq i}\left(J_{j}\right)$. The claim is a special case of [11, Corollary 6]. Let us show why. Given a term order $\sigma$ and two monomial vector spaces $A$ and $B$ of the same dimension, we set $A \geq_{\sigma} B$ if $A$ is generated monomials $c_{1}, \ldots, c_{k}, B$ is generated by monomials $d_{1}, \ldots, d_{k}$ where $c_{i}>_{\sigma} c_{i+1}, d_{i}>_{\sigma} d_{i+1}$ and $c_{i} \geq_{\sigma} d_{i}$ for all $i$. We have proved in [11, Corollary 6] that for every vector space $V$ of forms of a given degree and for any pair of term orders $\sigma$ and $\tau$ one has $\operatorname{Gin}_{\sigma}(V) \geq_{\sigma} \operatorname{Gin}_{\sigma}\left(\operatorname{in}_{\tau}(V)\right)$. In particular, 
if $V$ is in generic coordinates, then $\operatorname{in}_{\tau}(V)=\operatorname{Gin}_{\tau}(V)$ and by Lemma 2.3 we have $\operatorname{Gin}_{\sigma}\left(\operatorname{Gin}_{\tau}(V)\right)=\operatorname{Gin}_{\tau}(V)$. It follows that

$$
\operatorname{Gin}_{\sigma}(V) \geq_{\sigma} \operatorname{Gin}_{\tau}(V) .
$$

Taking $\sigma$ equal to revlex and $V=I_{j}$ we obtain the claim above.

More generally, the proof above shows that Theorem 5.1 also holds if $\operatorname{Gin}_{\tau}(I)$ is replaced by any Borel-fixed initial ideal of $I$. Theorem 5.1 should be compared with the example in [10] showing that, in general, there is no ideal with the smallest Betti numbers in the family of ideals with a given Hilbert function and with the example in [15, Section 6] showing that there is no ideal with the smallest Betti numbers in the family of Borel-fixed ideals with a given Hilbert function. Another application of [11, Corollary 6] easily yields:

Proposition 5.2. Among all the elements in $\operatorname{Gins}(I)$ the gin-lex is the closest to the lex-segment ideal of $I$, in the sense that if $L$ is the lex-segment ideal of $I, J$ is the gin-lex of $I$ and $H$ is any other gin of $I$, then $\operatorname{dim}(L \cap J)_{j} \geq \operatorname{dim}(L \cap H)_{j}$ for all $j$.

We know that the lex-segment ideal has the largest Betti numbers in the class of the ideals with a given Hilbert function and that in $\operatorname{Gins}(I)$ the gin-lex is the closest ideal to the lex-segment. Therefore it makes sense to ask whether the gin-lex has the largest Betti numbers among all the ideals in $\operatorname{Gins}(I)$. It turns out that (quite surprisingly) this is not the case in general. Even more interesting, there are ideals $I$ such that there is no ideal with the largest Betti numbers in $\operatorname{Gins}(I)$ and the gin-lex need not have maximal Betti numbers. The main difficulty in finding examples with these pathologies is that one must be able to detect all the gins of a given ideal which is a hard task. The examples we are going to present belong to a family of ideals called almost Borel-fixed. Let us define this family. Let $d \in \mathbf{N}$. Given a Borel-fixed space of monomials $A$ in $M_{d}$ and a monomial $b \in M_{d}$ we say that $b$ is a lower neighbor of $A$ if $b \notin A$ and $a \in A$ whenever $a>_{\text {Borel }} b$. Equivalently, $b$ is a lower neighbor of the Borel-fixed space $A$ if $b \notin A$ and $A+\langle b\rangle$ is Borel-fixed.

We denote by $\operatorname{Ln}(A)$ the set of the lower neighbor of $A$.

Definition 5.3. Let $A$ be a Borel-fixed space of monomials in $M_{d}$. Let $W$ be the vector space generated by the elements in $\operatorname{Ln}(A)$ and let $V \subseteq W$ be a subspace. The vector space $A+V$ is called an almost Borel-fixed space. A homogeneous ideal $I$ is said to be almost Borel-fixed if for each $d \in \mathbf{N}$ the space $I_{d}$ is almost Borel-fixed.

The main property of almost Borel-fixed spaces and ideals is that one has a complete description of the set of all the gins. We have:

Proposition 5.4. Let $A$ and $V$ be as in the Definition 5.3. Then for every term order $\tau$ one has

$$
\operatorname{Gin}_{\tau}(A+V)=A+\operatorname{in}_{\tau}(V) .
$$

Proof. The left- and right-hand sides of the equality we have to prove are vector spaces of the same dimension. Therefore it is enough to prove the inclusion $\supseteq$. Since $A+V \supseteq A$ we have $\operatorname{Gin}_{\tau}(A+V) \supseteq \operatorname{Gin}_{\tau}(A)=A$. To conclude the proof we need to show that for a generic $g \in \mathrm{GL}_{n}(K)$ and for all $f \in V$ one has $\operatorname{in}_{\tau}(f) \in$ $\operatorname{in}_{\tau}(g(A+V))$. Let $m$ be a lower neighbor of $A$. Note that $A$ and $A+\langle m\rangle$ are Borel-fixed spaces. Then, by virtue of Lemmas [2.3] and [2.4, $\operatorname{in}_{\tau}(g(A))=A$ and the 
normal form of $g(m)$ with respect to $g(A)$ has the form $m+h$, where $h$ contains only monomials which are smaller (in the Borel order) then $m$. Now say $f=\lambda_{1} m_{1}+$ $\cdots+\lambda_{r} m_{r}$, where the $m_{i}$ are lower neighbors of $A$ and $\lambda_{i} \in K$. It follows that the normal form of $g(f)$ with respect to $g(A)$ is $f+H$ and each monomial in $H$ is smaller in the Borel order than some of the $m_{i}$. This implies that $\operatorname{in}_{\tau}(f+H)=\operatorname{in}_{\tau}(f)$ and hence that $\operatorname{in}_{\tau}(f) \in \operatorname{in}_{\tau}(g(A+V))$.

Since $\operatorname{Gin}_{\tau}(I)=\bigoplus_{d} \operatorname{Gin}_{\tau}\left(I_{d}\right)$, Proposition 5.4 allows us to describe all the gins of an almost Borel-fixed ideal provided, of course, we have a description of the decomposition of $I_{d}$ as " $A+V$ " for each $d$. Note however that if $U$ is an almost Borel-fixed space, then $U R_{1}$ need not be almost Borel-fixed; see the next example.

Example 5.5. a) The simplest almost Borel-fixed space (which is not Borel-fixed) is the following: in 3 variables, $A=\left\langle x_{1}^{2}, x_{1} x_{2}\right\rangle, \operatorname{Ln}(A)=\left\{x_{1} x_{3}, x_{2}^{2}\right\}$ and $V=$ $\left\langle x_{1} x_{3}+x_{2}^{2}\right\rangle$. Then, according to Proposition 5.4 the almost Borel-fixed space $U=A+V$ has only two distinct gins, the gin-revlex $A+\left\langle x_{2}^{2}\right\rangle$ and the gin-lex $A+\left\langle x_{1} x_{3}\right\rangle$.

b) If we embed the example of part a) in a ring with an extra variable $x_{4}$, then it is still almost Borel-fixed but it is easy to see that $R_{1} U$ is not almost Borel-fixed.

Construction 5.6. One can construct an almost Borel-fixed ideal as follows: Let $T$ be a set of Borel-incomparable elements in $M_{d}$. Set

$$
\begin{aligned}
& X=\left\langle n \in M_{d}: \text { there exists } m \in T \text { such that } n \geq_{\text {Borel }} m\right\rangle, \\
& A=\left\langle n \in M_{d}: \text { there exists } m \in T \text { such that } n>_{\text {Borel }} m\right\rangle .
\end{aligned}
$$

Let $f_{1}, \ldots, f_{p}$ be polynomials with disjoint supports such that each $f_{i}$ is a sum of elements in $T$. Let $B$ be a Borel-fixed subspace of $M_{d+1}$ such that $B$ contains $X R_{1}$ (e.g. $B=X R_{1}$ or $\left.B=M_{d+1}\right)$. Then the ideal $I=(A)+\left(f_{1}, \ldots, f_{p}\right)+(B)$ is almost Borel-fixed and

$$
\operatorname{Gin}_{\tau}(I)=(A)+\left(\operatorname{in}_{\tau}\left(f_{1}\right), \ldots, \operatorname{in}_{\tau}\left(f_{p}\right)\right)+(B) .
$$

One can iterate the construction by taking a set of incomparable elements in $M_{d+1} \backslash$ $B$ and so on to get an almost Borel-fixed ideal which is non-Borel fixed in more than one degree.

Example 5.7. Applying Construction [5.6] in $K\left[x_{1}, x_{2}, x_{3}, x_{4}\right]$ to $T=\left\{x_{1} x_{3}^{2}, x_{2}^{2} x_{4}\right\}$ with $p=1, f=x_{1} x_{3}^{2}+x_{2}^{2} x_{4}$, and $B=X R_{1}$, one gets

$$
A=\left\langle x_{1}^{3}, x_{1}^{2} x_{2}, x_{1}^{2} x_{3}, x_{1}^{2} x_{4}, x_{1} x_{2}^{2}, x_{1} x_{2} x_{3}, x_{1} x_{2} x_{4}, x_{2}^{3}, x_{2}^{2} x_{3}\right\rangle
$$

and the ideal

$$
I=(A)+(f)+\left(x_{2}^{2} x_{4}^{2}\right) .
$$

The ideal $I$ is almost Borel-fixed and has exactly two gins. The gin-lex (which is also the gin-revlex in this case) is

$$
G_{1}=(A)+\left(x_{1} x_{3}^{2}, x_{2}^{2} x_{4}^{2}\right)
$$

and the gin with respect to the term order induced, for instance, by the weight function $(6,5,2,1)$ is

$$
G_{2}=(A)+\left(x_{2}^{2} x_{4}, x_{1} x_{3}^{3}, x_{1} x_{3}^{2} x_{4}\right)
$$


The Macaulay diagrams of the Betti numbers of $G_{1}$ and $G_{2}$ are:

$$
G_{1}: \begin{array}{|c|c|c|c|}
\hline 10 & 17 & 10 & 2 \\
\hline 1 & 3 & 3 & 1 \\
\hline
\end{array}
$$

$G_{2}:$\begin{tabular}{|c|c|c|c|}
\hline 10 & 18 & 12 & 3 \\
\hline 2 & 5 & 4 & 1 \\
\hline
\end{tabular}

This shows that the gin-lex need not have the largest Betti numbers among all the gins of a given ideal.

In order to describe an ideal $I$ such that in $\operatorname{Gins}(I)$ there is no ideal with largest Betti numbers, we need to enlarge a little the ambient space, i.e. we need more variables and higher degree monomials.

Example 5.8. Applying Construction [5.6] in $K\left[x_{1}, \ldots, x_{7}\right]$ to

$$
T=\left\{x_{1} x_{3} x_{6}^{2}, x_{2}^{2} x_{3} x_{7}, x_{1} x_{4}^{2} x_{6}, x_{2}^{2} x_{4}^{2}\right\}
$$

with $p=2, f_{1}=x_{1} x_{3} x_{6}^{2}+x_{2}^{2} x_{3} x_{7}$ and $f_{2}=x_{1} x_{4}^{2} x_{6}+x_{2}^{2} x_{4}^{2}$, and $B=X R_{1}$, one gets

$A=\left\langle x_{1}^{4}, x_{1}^{3} x_{2}, x_{1}^{3} x_{3}, x_{1}^{3} x_{4}, x_{1}^{3} x_{5}, x_{1}^{3} x_{6}, x_{1}^{3} x_{7}, x_{1}^{2} x_{2}^{2}, x_{1}^{2} x_{2} x_{3}, x_{1}^{2} x_{2} x_{4}, x_{1}^{2} x_{2} x_{5}\right.$,

$x_{1}^{2} x_{2} x_{6}, x_{1}^{2} x_{2} x_{7}, x_{1}^{2} x_{3}^{2}, x_{1}^{2} x_{3} x_{4}, x_{1}^{2} x_{3} x_{5}, x_{1}^{2} x_{3} x_{6}, x_{1}^{2} x_{3} x_{7}, x_{1}^{2} x_{4}^{2}, x_{1}^{2} x_{4} x_{5}, x_{1}^{2} x_{4} x_{6}$, $x_{1}^{2} x_{5}^{2}, x_{1}^{2} x_{5} x_{6}, x_{1}^{2} x_{6}^{2}, x_{1} x_{2}^{3}, x_{1} x_{2}^{2} x_{3}, x_{1} x_{2}^{2} x_{4}, x_{1} x_{2}^{2} x_{5}, x_{1} x_{2}^{2} x_{6}, x_{1} x_{2}^{2} x_{7}, x_{1} x_{2} x_{3}^{2}$, $x_{1} x_{2} x_{3} x_{4}, x_{1} x_{2} x_{3} x_{5}, x_{1} x_{2} x_{3} x_{6}, x_{1} x_{2} x_{3} x_{7}, x_{1} x_{2} x_{4}^{2}, x_{1} x_{2} x_{4} x_{5}, x_{1} x_{2} x_{4} x_{6}, x_{1} x_{2} x_{5}^{2}$, $x_{1} x_{2} x_{5} x_{6}, x_{1} x_{2} x_{6}^{2}, x_{1} x_{3}^{3}, x_{1} x_{3}^{2} x_{4}, x_{1} x_{3}^{2} x_{5}, x_{1} x_{3}^{2} x_{6}, x_{1} x_{3} x_{4}^{2}, x_{1} x_{3} x_{4} x_{5}, x_{1} x_{3} x_{4} x_{6}$, $x_{1} x_{3} x_{5}^{2}, x_{1} x_{3} x_{5} x_{6}, x_{1} x_{4}^{3}, x_{1} x_{4}^{2} x_{5}, x_{2}^{4}, x_{2}^{3} x_{3}, x_{2}^{3} x_{4}, x_{2}^{3} x_{5}, x_{2}^{3} x_{6}, x_{2}^{3} x_{7}, x_{2}^{2} x_{3}^{2}, x_{2}^{2} x_{3} x_{4}$, $\left.x_{2}^{2} x_{3} x_{5}, x_{2}^{2} x_{3} x_{6}\right\rangle$ and

$$
I=(A)+\left(f_{1}, f_{2}\right)+\left(x_{1} x_{4}^{2} x_{6} x_{7}, x_{2}^{2} x_{3} x_{7}^{2}, x_{1} x_{4}^{2} x_{6}^{2}\right) .
$$

The ideal $I$ is almost Borel-fixed and has exactly 3 gins: the gin-revlex

$$
G_{1}=(A)+\left(x_{2}^{2} x_{4}^{2}, x_{1} x_{3} x_{6}^{2}\right)+\left(x_{1} x_{4}^{2} x_{6} x_{7}, x_{2}^{2} x_{3} x_{7}^{2}, x_{1} x_{4}^{2} x_{6}^{2}\right),
$$

the gin-lex

$$
G_{2}=(A)+\left(x_{1} x_{4}^{2} x_{6}, x_{1} x_{3} x_{6}^{2}\right)+\left(x_{2}^{2} x_{4}^{2} x_{7}, x_{2}^{2} x_{3} x_{7}^{2}, x_{2}^{2} x_{4}^{2} x_{6}, x_{2}^{2} x_{4}^{2} x_{5}, x_{2}^{2} x_{4}^{3}\right)
$$

and the gin w.r.t. to the term order induced by the weight $(7,6,5,4,3,2,1)$

\begin{tabular}{|c|c|c|c|c|c|c|c|}
\hline \multirow{2}{*}{$G_{1}:$} & 64 & 240 & 397 & 363 & 190 & 53 & 6 \\
\hline & 3 & 17 & 40 & 50 & 35 & 13 & 2 \\
\hline & 64 & 242 & 404 & 372 & 195 & 54 & 6 \\
\hline & 5 & 24 & 49 & 55 & 36 & 13 & 2 \\
\hline \multirow{2}{*}{$7_{3}:$} & 64 & 241 & 402 & 373 & 200 & 58 & 7 \\
\hline & 4 & 22 & 50 & 60 & 40 & 14 & 2 \\
\hline
\end{tabular}

$$
G_{3}=(A)+\left(x_{2}^{2} x_{4}^{2}, x_{2}^{2} x_{3} x_{7}\right)+\left(x_{1} x_{4}^{2} x_{6} x_{7}, x_{1} x_{3} x_{6}^{2} x_{7}, x_{1} x_{4}^{2} x_{6}^{2}, x_{1} x_{3} x_{6}^{3}\right) .
$$

Here the crucial observation is that there are no term orders such the in $\left(f_{1}\right)=$ $x_{2}^{2} x_{3} x_{7}$ and $\operatorname{in}\left(f_{2}\right)=x_{1} x_{4}^{2} x_{6}$. The Macaulay diagrams of the Betti numbers of $G_{1}, G_{2}$ and $G_{3}$ are: 
Therefore there is no gin with the largest Betti numbers since $G_{2}$ and $G_{3}$ have maximal and incomparable Betti diagrams.

A slight variation in the construction above also allows us to describe ideals such that the gin-lex does not even have the largest Castelnuovo-Mumford regularity among all the gins. One may ask whether ideals with few generators can behave as the ideals of Examples 5.7 and 5.8. There is experimental evidence that even a monomial ideal with 2 generators can have these behaviors. For instance, we have run $\mathrm{CoCoA}$ to get the gins of the ideal $I=\left(x_{1}^{4}, x_{2}^{2} x_{3} x_{4}\right)$. The computations show that the gin-lex of $I$ does not have maximal Betti numbers and that the there seems to be 351 gins with 3 different sets of maximal Betti numbers. The problem with these computations is twofold: first when we compute $\operatorname{in}_{\tau}(g(I))$ by taking a random (upper triangular) matrix $g$, what we get is $\operatorname{Gin}_{\tau}(I)$ but only "with high probability", and, second, it is not clear how can we be sure that the list of the gins is complete.

Let us conclude the section by showing that:

Proposition 5.9. An almost Borel-fixed ideal is componentwise linear.

Proof. Let $\tau$ be the revlex order. Let $A, V$ be as in Definition 5.3. Let $f_{1}, \ldots, f_{p}$ be generators of $V$. It is harmless to assume that the monomials $\operatorname{in}_{\tau}\left(f_{i}\right)$ are distinct. Let $D$ be the set of the monomials in $A$ and set $G=D \cup\left\{f_{1}, \ldots, f_{p}\right\}$. Let $I$ be the ideal generated by the set $G$. We have to show that $I$ has a linear resolution. Set $m_{i}=\operatorname{in}_{\tau}\left(f_{i}\right)$ and let $J$ be the ideal generated by $D$ and by $m_{1}, \ldots, m_{p}$. Note that $J$ has a linear resolution since it is a Borel-fixed ideal generated in a single degree. Therefore it suffices to show that $\operatorname{in}_{\tau}(I)=J$ or, equivalently, that $G$ is a Gröbner basis. We apply Buchberger's algorithm to $G$. $\operatorname{Set}_{\tau}(G)=D \cup\left\{m_{1}, \ldots, m_{p}\right\}$. It suffices to show that any $S$-polynomial which corresponds to a minimal syzygy among elements of $\operatorname{in}_{\tau}(G)$ reduces to 0 via $G$. The elements of $\operatorname{in}_{\tau}(G)$ generate a Borel-fixed space. Their minimal syzygies are described by the Eliahou-Kervaire resolution. They have the following form: for every term $n \in \operatorname{in}_{\tau}(G)$ and any $j<i=\max (n)$ set $n_{1}=n x_{j} / x_{i}$. Then $n_{1} \in \operatorname{in}_{\tau}(G)$ and $x_{j} n-x_{i} n_{1}=0$ is a minimal syzygy. If $n \in D$, then $n_{1} \in D$ as well and the corresponding $S$-polynomial is 0 as any $S$-polynomial among monomials. If instead $n \notin D$, then $n=m_{k}$ for some $k$, say $k=1$. In this case $n_{1}$ must be in $D$ since by construction $n_{1}>_{\text {Borel }} n$ and, by the very definition of almost Borel-fixed space, $n$ is a lower neighbor of $A$. So the $S$-polynomial corresponding to the given syzygy is $S=x_{j} f_{1}-x_{i} n_{1}$. Let $b$ be a non-initial monomial of $f_{1}$ and set $u=\max (b)$. Then $i=\max \left(m_{1}\right) \leq \max (b)=u$ (here we use the fact that the term order is revlex) and the $x_{j} b=x_{u} b_{1}$ with $b_{1}=x_{j} a / x_{u} \in D$ since $b$ is also a lower neighbor of $A$ and $b_{1}>_{\text {Borel }} b$. This implies that $S$, being a sum of multiples of monomials of $D$, reduces to 0 via $G$.

Note that, with the notation of the proof of Proposition 5.9, we did not prove that $\operatorname{Gin}(I)=J$, just that $\operatorname{in}(I)=J$. But since, a posteriori, we know that $I$ is componentwise linear, then its gin-revlex must be generated in the initial degree. Then it follows from Proposition 5.4 that $\operatorname{Gin}(I)=J$. Simple examples show that, in general, the non-revlex gins of $I$ might need generators in higher degrees.

\section{Some Questions}

We have shown that, passing to a generic initial ideal, the Koszul-Betti numbers can only increase. Most likely the same is true for any initial ideal. 
Question 6.1. Is it true that $\beta_{i j p}(R / I) \leq \beta_{i j p}\left(R / \operatorname{in}_{\tau}(I)\right)$ for all $i, j, p$ and for all term orders $\tau$ ?

How much of what we have shown can be extended to positive characteristic? For instance:

Question 6.2. Do Theorem 4.3(b) and Theorem 5.1 hold if the base field has positive characteristic?

We have seen that the gin-lex need not have the largest Betti numbers among all the gins of a given ideal unless it should happen to be a lex-segment ideal. On the other hand, the gin-lex is very rarely equal to the lex-segment, even for ideals generated by generic polynomials. For instance the gin-lex of two generic quadrics in four variables differs from the lex-segment already in degree 4. However there is experimental evidence that the answer to the following questions might be positive:

Question 6.3. Let $I$ be the ideal generated by $m$ generic forms of degree $d$ in $n$ variables with $m \geq n$. Is it true that the gin-lex of $I$ equals the lex-segment?

Question 6.4. Let $I$ be an ideal generated by generic forms of given degrees. Does the gin-lex have the largest Betti numbers among all the gins of $I$ ?

Note that the set Gins $(I)$ can be very large even for "small" ideals. For instance in four variables, for two generic cubics we have detected 93 distinct gins (and most likely there are no others) while for two generic quartics we have detected more than 3000 gins (and most likely there are many others). In these cases we have checked that the gin-lex have the largest Betti numbers among all the gins we have found.

In view of Theorems 1.2 and 1.3, one could ask what happens if we assume that $\beta_{i}(R / I)=\beta_{i}(R / \operatorname{Gin}(I))$ or $\beta_{i}(R / I)=\beta_{i}(R / \operatorname{Lex}(I))$ for some $i>0$. We cannot conclude that all the Betti numbers are equal. This is because some Betti numbers (typically the last) can be forced by the Hilbert function. For instance all the rings with Hilbert function 1,3,4,0 have the same last Betti number but the rest of the resolution can vary. On the other hand there might be some sort of rigidity toward the end of the resolution and one can ask the following:

Question 6.5. Let $I$ be a homogeneous ideal and $J$ be either $\operatorname{Gin}(I)$ or $\operatorname{Lex}(I)$. Assume that $\beta_{i}(R / I)=\beta_{i}(R / J)$ for some $i>0$. Does this imply that $\beta_{j}(R / I)=$ $\beta_{j}(R / J)$ for all $j \geq i$ ?

After this paper was written Giulio Caviglia showed that Question 6.1 has a positive answer; see [8]. Furthermore, in collaboration with Herzog and Hibi, we showed that the rigidity question, Question [6.5 has also a positive answer (in characteristic 0 ).

\section{REFERENCES}

1. A. Aramova, J. Herzog, Koszul cycles and Eliahou-Kervaire type resolutions, J. Algebra 181 (1996), no. 2, 347-370. MR 97c:13009

2. A. Aramova, J. Herzog, Almost regular sequences and Betti numbers, Amer. J. Math. 122 (2000), no. 4, 689-719. MR 2001i:13029

3. A. Aramova, J. Herzog, T. Hibi, Ideals with stable Betti numbers, Adv. Math. 152 (2000), no. 1, 72-77. MR 2001d:13012

4. A. Bigatti, Upper bounds for the Betti numbers of a given Hilbert function, Comm. Algebra 21 (1993), no. 7, 2317-2334. MR 94c:13014 
5. D. Bayer, The division algorithm and the Hilbert scheme, Ph.D. Thesis, Harvard University, June 1982, 168 pages.

6. D. Bayer, M. Stillman, A criterion for detecting m-regularity, Invent. Math. 87 (1987), no. 1, 1-12. MR 87k:13019

7. W. Bruns, J. Herzog, Cohen-Macaulay rings, Cambridge Studies in Advanced Mathematics, 39. Cambridge University Press, Cambridge, 1993. MR 95h:13020

8. G. Caviglia, , Ph.D. Thesis, in preparation (2004).

9. A. Capani, G. Niesi, L. Robbiano, CoCoA, a system for doing Computations in Commutative Algebra, Available from cocoa.dima.unige.it.

10. H. Charalambous, G. Evans, Resolutions with a given Hilbert function, Commutative algebra: syzygies, multiplicities, and birational algebra (South Hadley, MA, 1992), 19-26, Contemp. Math., 159, Amer. Math. Soc., Providence, RI, 1994. MR 95a:13021

11. A. Conca, Reduction numbers and initial ideals, preprint 2001, Proc. Amer. Math. Soc. 131 (2003), 1015-1020.

12. A. Conca, J. Herzog, T. Hibi, Rigid resolutions and big Betti numbers, preprint 2003, in preparation.

13. D. Eisenbud, Commutative algebra. With a view toward algebraic geometry, Graduate Texts in Mathematics, 150. Springer-Verlag, New York, 1995. MR 97a:13001

14. S. Eliahou, M. Kervaire, Minimal resolutions of some monomial ideals, J. Algebra 129 (1990), no. 1, 1-25. MR 91b:13019

15. C. Francisco, Minimal graded Betti numbers and stable ideals, Comm. Algebra 28 (2000), no. 3, 1515-1531. MR 2001a:13021

16. G. Golub, C. Van Loan, Matrix computations, Second edition. Johns Hopkins Series in the Mathematical Sciences, 3. Johns Hopkins University Press, Baltimore, MD, 1989, xxii+642 pp. MR 90d:65055

17. M. Green, Generic initial ideals, Six lectures on commutative algebra (Bellaterra, 1996), 119-186, Progr. Math., 166, Birkhäuser, Basel, 1998. MR 99m:13040

18. M. Green, M. Stillman, A tutorial on generic initial ideals, Gröbner bases and applications (Linz, 1998), 90-108, London Math. Soc. Lecture Note Ser., 251, Cambridge Univ. Press, Cambridge, 1998. MR 2000g:13019

19. J. Herzog, T. Hibi, Componentwise linear ideals, Nagoya Math. J. 153 (1999), 141-153. MR 2000i:13019

20. J. Herzog, A. Simis, W. Vasconcelos, Approximation complexes of blowing-up rings. II, J. Algebra 82 (1983), no. 1, 53-83. MR 85b:13015

21. H. Hulett, Maximum Betti numbers of homogeneous ideals with a given Hilbert function, Comm. Algebra 21 (1993), no. 7, 2335-2350. MR 94c:13015

22. M. Kreuzer, L. Robbiano, Computational commutative algebra 1, Springer-Verlag, Berlin, 2000, x+321 pp. MR 2001j:13027

23. K. Pardue, Non-standard Borel-fixed ideals, Ph.D. Thesis, Brandeis University, May 1994, 69 pages.

24. K. Pardue, Deformation classes of graded modules and maximal Betti numbers, Illinois J. Math. 40 (1996), no. 4, 564-585. MR 97g:13029

25. E. Sbarra, Upper bounds for local cohomology for rings with given Hilbert function, Comm. Algebra 29 (2001), no. 12, 5383-5409. MR 2002j:13024

26. B. Sturmfels, Gröbner bases and convex polytopes, University Lecture Series, 8. American Mathematical Society, Providence, RI, 1996, xii+162 pp. MR 97b:13034

27. G. Valla, Problems and results on Hilbert functions of graded algebras. Six lectures on commutative algebra (Bellaterra, 1996), 293-344, Progr. Math., 166, Birkhäuser, Basel, 1998. MR 99k:13022

Dipartimento di Matematica, Universita' di Genova, Genova, I-16146 Italy

E-mail address: conca@dima.unige.it 